Trauma Berufskrankh $2012 \cdot$ 14[Suppl 2]:230-235 DOI 10.1007/s10039-011-1756-6

Online publiziert: 5. August 2011

(c) Springer-Verlag 2011
V. Kaiser

Grafenau

\section{Beckenfrakturen und hüftgelenknahe Verletzungen}

\section{Überlegungen zur MdE-Bewertung}

\section{Vorbemerkung}

Ein allgemeines Unbehagen hinsichtlich der Tabellenwerte zur Minderung der Erwerbsfähigkeit (MdE), die in der gesetzlichen Unfallversicherung bei den Begutachtungen und Rentenleistungen umgesetzt werden, hat eine lange Tradition. Seit Einführung dieses gesetzlichen Instruments bzw. Rechtsbegriffs, mit Bezug auf ärztlich-medizinische Sachverhalte, wird insbesondere auch von der Rechtsprechung darauf hingewiesen, dass die MdESätze laufend überprüft und den Entwicklungen auf dem Feld der Bemessungskriterien (Arbeitsmarktbedingungen usw.) angepasst werden müssen [z. B. BSG (Bundessozialgericht) vom 26.06.1985, SozR (Sozialrecht) $2200 \$ 581$ Nr. 23, [4, 12, 15, 21, 26]]. Abgesehen von einigen Berufskrankheiten gibt es aber bis heute im Wesentlichen nur singuläre Änderungsvorschläge, was nicht zuletzt an den Schwierigkeiten und dem Arbeitsaufwand bei einer exakten Beachtung der genau genommenen Gesetzeslage liegt.

In der Kommission Gutachten der Deutschen Gesellschaft für Orthopädie und Unfallchirurgie (DGOU), in der auch Juristen bzw. Verwaltungsfachleute mitarbeiten, war eine breitere Überprüfung der aus der Praxis bekannten MdE-Werte und v. a. der in der Literatur veröffentlichten Tabellenwerke bereits vor einiger Zeit angesprochen worden. Damals wurde je- doch ein solches Vorhaben wegen gesetzespolitischer Überlegungen, das Rentensystem der gesetzlichen Unfallversicherung und auch die Rechtsfigur der MdE umzugestalten, nicht konkret aufgegriffen. Zwischenzeitlich wurden jedoch im Gesetz zur Modernisierung der gesetzlichen Unfallversicherung (Unfallversicherungsmodernisierungsgesetz - UVMG) vom 04.11.2008 die bisherigen Regelungsstrukturen beibehalten, sodass in absehbarer Zeit insoweit keine entscheidenden Änderungen zu erwarten sind. Als aus dem Mitgliederkreis der DGOU erneut eine Analyse dieses wichtigen Begutachtungskomplexes angeregt wurde, entschied sich die Kommission Gutachten für die Durchführung eines solch aufwendigen Projekts.

Dieses MdE-Projekt wird trotz des beschränkten Titels dieses Programmteils der Unfallmedizinischen Tagung - Verletzungen des Beckens und der Hüfte - näher dargestellt, um den Kontext der Überlegungen speziell zur MdE bei Beckenverletzungen transparent zu machen. Der Gesamtkomplex ist wiederum für die allgemeine Einschätzung der hier thematisierten MdE-Vorschläge von Bedeutung.

\section{Äußeres Erscheinungsbild der allgemeinen MdE-Praxis}

Der erste, kursorische Befund der veröffentlichten bzw. allgemein bekannt gewor- denen Zusammenstellungen von MdEWerten und zugänglich gemachten einzelnen MdE-Einschätzungen weist zwar verschiedene Facetten auf und ergibt kein einheitliches Bild. Zunächst deutet er aber nicht auf einen dringenden Handlungsbedarf für eine kritische Analyse der MdEWerte hin, jedenfalls nicht mit einer umfassenden und über singuläre Auffälligkeiten hinausgehenden Ausrichtung:

Eine kleinere Zahl der weithin bekannten MdE-Werte ist mit einer gefestigten Anwendungspraxis und überzeugender sachlicher Begründung etabliert. Manche Eck- bzw. Grundwerte und Bemessungsorientierungen (z. B. Verlust des Daumens und die Gleichbewertung von rechter und linker Hand bzw. Gebrauchs- und Beihand) werden dabei als allgemeine oder (allgemein) anerkannte Erfahrungswerte bezeichnet, die in der Form von qualifizierten Erfahrungssätzen - nach Literaturmeinungen $[11,13,14$, 16] und Andeutungen in der Rechtsprechung [BSGE (Entscheidungen des Bundessozialgerichts) 40,120; BSGE 82,212; BSG Breithaupt 2001, 783] - rechtlich in einem gegenüber einfachen Erfahrungssätzen erhöhtem Maß beachtet werden müssen; zumindest können sie als eine Art verbreiteter Standard angesehen werden. Die besondere, gesteigerte Rechtsqualität eines sogenannten antizipierten Sachverständigengutachtens im Sinne vorweggenommener Beurteilungen maß 
die BSG-Rechtsprechung bisher nur (wenigen) MdE-Tabellenwerken für Berufskrankheiten zu [z. B. Königsteiner Merkblatt für die Lärmschwerhörigkeit (BSG Breithaupt 2001, 783)].

Insbesondere jedoch wird das aktuelle MdE-Erscheinungsbild von den in letzter Zeit verstärkten Aktivitäten zu aufwendigeren Konsensverfahren geprägt, die den angestrebten MdE-Empfehlungen eine größere Autorität verleihen können. Solche Anstöße kommen vorwiegend aus einem einschlägigen medizinischen Fachgebiet und für dessen allgemeines wissenschaftlich-berufliches Anliegen; daneben bemühen sich die Versicherer, insbesondere für neue versicherte Gesundheitsschädigungen bzw. -risiken, zusammen mit der maßgeblichen ärztlichen Kompetenz um Abstimmungen bzw. Übereinkünfte zu den betreffenden MdE-Einschätzungen (z. B. im Bereich der Berufskrankheiten, wie u. a. dem Falkensteiner Merkblatt für Atemwegserkrankungen). Abgesehen davon lässt sich im allgemeinen Erfahrungsbild eine gewisse Bewegung mit neuen MdE-Vorschlägen v. a. in der Literatur und innerhalb bestimmter Fachkreise sowie bei der gerichtlichen Praxis, insbesondere der Untergerichte, feststellen (z. B. für psychische Störungen [6]).

Abgesehen von ihrem ersten Grundzug führte diese Entwicklung, die wiederum nur für einzelne Bereiche von Gesundheitsschäden festgestellt werden kann, jedoch bisher nicht zu wesentlichen Übereinstimmungen und zu einer allgemeinen Akzeptanz oder gar verfestigten Anwendungspraxis. Vielmehr wird das Gesamtbild insoweit immer noch durch z. T. markant unterschiedliche MdEWerte geprägt. Speziell für die Literatur ist kennzeichnend, dass nähere Begründungen fehlen, und die Vorschläge auch sonst nicht nachvollziehbar sind. Ein näherer Vergleich von Tabellen-Werken lässt zudem manchmal vermuten, dass bereits früher zu reflektierende MdE-Sätze unkritisch übernommen bzw. weitergeschrieben wurden. Auffällig sind hier nicht zuletzt vielfach eine große Spreizung bzw. ein breiter MdE-Rahmen, bei verhältnismäßig wenigen Werten, die für die Einschätzung im individuellen Fall einen groBen Spielraum zulassen und keine Gleich- behandlung der Versicherten usw. gewährleisten. Im Übrigen gelten auch gängige MdE-Sätze nicht ewig, wenngleich ihre Überprüfung nicht an vorderster Stelle steht.

\section{Bedeutsame Entwicklungen hinsichtlich der gesetzlichen MdE-Kriterien}

Bereits das vielbeschriebene Kennzeichen unserer Epoche der Moderne und ihren sensiblen Aktualisierungen (als Spät-, Post-, Postpostmoderne usw.), eine allgemeine und auch im Einzelnen beobachtete Beschleunigung unserer Lebensumstände [7, 23], vermag eine Überprüfung der MdE-Werte veranlassen. Jedenfalls wird eine erste Evidenz für ein solches Vorhaben durch nähere Feststellungen des rechtlich beachtlichen Kontextes, und zwar in mehrfacher Hinsicht, bestätigt. Im Wesentlichen sind es 3 umfassende Entwicklungslinien, die sich hierbei herauskristallisieren und die Gesetzesmäßigkeit vieler praktizierter MdE-Bewertungen in Frage stellen können.

\section{Gerichtliche Urteile}

Mit einer intensiveren höchstrichterlichen Spruchpraxis zur Rentenleistung in der gesetzlichen Unfallversicherung wurden auch rechtliche Grundsätze und Einzelmaßstäbe der MdE-Bewertung konkretisiert und ausdifferenziert. Dazu lässt sich beispielhaft, neben der Anforderung eines grundsätzlichen 3-stufigen Einschätzungsverfahren bei der einzelnen MdE-Feststellung, die Rechtsprechung des Bundessozialgerichts anführen, dass sich besondere und selbst erst neuere (gesicherte und nachhaltige) Therapieerfolge prinzipiell auf den MdE-Grad auswirken können (u. a. mit bestimmten Implantaten oder bei der Einnahme von Medikamenten, bereits BSGE 28,41, [1, 9, 20]).

\section{Veränderung der Arbeitsanforderungen}

Im Hinblick auf den gesetzlichen MdEBegriff [ $\$ 56$ SGB (Sozialgesetzbuch) Abs. 2 VII], der auf die (mit den Funktionsbeeinträchtigungen) verbliebenen Arbeitsmöglichkeiten („auf dem gesamten Ge- biet des Erwerbslebens") abstellt, werden die MdE-Werte auch maßgeblich von den Wandlungen der Berufswelt und des allgemeinen Arbeitsmarkts beeinflusst. Von Bedeutung sind neben der grundlegenden praktischen Umstrukturierung ganzer Berufsfelder (z. B. im handwerklichen Bereich) insbesondere generell und erheblich veränderte Arbeitsbedingungen. Letztere Facette zeigt sich symptomatisch bei den Verhältnissen in der Industrie, mit der aktuellen Produktionsweise sowie der modernen Gestaltung der Arbeitsorganisation und fortschrittlichen Ausstattung der einzelnen Arbeitsplätze (z. B. in den Autofabriken). Sie findet sich aber auch in kleineren Werkstätten und z. B. auf Baustellen. Markante Auswirkungen dieser Entwicklung sind eine durchgreifende Verringerung der körperlichen Schwerarbeit, hauptsächlich des beschwerlichen Hebens und Tragens, und die Zunahme sitzender Tätigkeiten bei einer Steigerung leichter manueller Arbeitsverrichtungen.

\section{Fortschritte der Medizin}

Von grundsätzlicher rechtlicher Relevanz, wie auch die oben angeführte Rechtsprechung zeigt, sind in diesem Kontext auch die großen Fortschritte in der medizinischen Therapie, einschließlich der prothetischen Versorgungsmöglichkeiten und Verfügbarkeit von (Funktionsbeeinträchtigungen vermindernden oder ausgleichenden) Hilfsmitteln. Mit dem besonderen Zuständigkeitsbereich der gesetzlichen Unfallversicherung kommt hierbei der modernen Wiederherstellungschirurgie und ihrem erreichten Behandlungsstandard sowie den optimierten, spezialisierten Rehabilitationsverfahren ein entscheidendes Gewicht zu; einen großen Anteil daran hat nicht zuletzt der hohe Stand der heutigen Prothesentechnik.

\section{MdE-Projekt der DGOU - Kommission Gutachten}

Nach dem groben Design des gegenständlichen MdE-Projekts geht es zunächst darum, die in der Literatur und Begutachtungs- bzw. Leistungspraxis festzustellenden MdE-Werte - im möglichen Rahmen - zu untersuchen (Istanalyse). Demgemäß beschränkt sich das Vorhaben auf die we- 
sentlichen und auf dem unfallchirurgischorthopädischen Fachgebiet liegenden Gesundheitsschäden bzw. Funktionsbeeinträchtigungen, sodass der Bereich der Berufskrankheiten generell ausgespart wird.

Hauptsächlich kommt es auf 2 Perspektiven an:

- Inwieweit kann einzelnen MdE-Werten bzw. MdE-Gruppen die Qualität allgemein anerkannter Standardwerte, auch als allgemeine Erfahrungswerte im Sinne der Rechtsprechung, beigemessen werden?

- Welche MdE-Werte, v. a. so weit sie nicht einen solchen Rang einnehmen, entsprechen (noch) der gesetzlichen Funktion und Zielsetzung des Rechtsinstruments der MdE?

Falls für gängige MdE-Sätze eine aktuelle Gesetzesmäßigkeit fraglich erscheint, sollen in einer 2. Projektstufe materiell gesicherte Werte erarbeitet und dabei auch neue, von bisherigen Bemessungspraktiken bzw. Literaturmeinungen abweichende Vorschläge entwickelt werden. Mit dem Kommissionskonsens wird im Weiteren eine breite Diskussion in den betreffenden Fachkreisen und Anwendungsbereichen angestrebt, um übergreifende inhaltliche Konventionen zu erreichen. Dazu kann auch ein Leitlinienverfahren eingesetzt werden, und zwar nach dem Modell der gesetzlichen Unfallversicherung [gemäß Abstimmung zwischen DGUV (Deutsche Gesetzliche Unfallversicherung) und AWMF (Arbeitsgemeinschaft der Wissenschaftlichen Medizinischen Fachgesellschaften e.V.)]. Zielvorstellung des Gesamtprojekts ist, über eine große Akzeptanz v. a. der Vorschläge mit neuen, geänderten MdE-Werte die Weiterentwicklung zu rechtlich qualifizierten allgemeinen Erfahrungswerten zu ermöglichen.

Der Verfahrensablauf des MdE-Projekts ergibt sich aus dem umfänglichen Arbeitsvolumen, der größeren Mitgliederzahl der (gesamten) Kommission Gutachten und deren vielfältigen, laufenden Themen bzw. Aktivitäten: Der umfassende Komplex wird in einer kleinen $M d E$ Arbeitsgruppe aus ärztlichen Gutachtern und Juristen (mit verwaltungsseitigem und richterlichem Hintergrund) aufbereitet. Die vorläufig erarbeiteten Ergebnisse werden laufend in die Kommission ein-

Trauma Berufskrankh 2012 - 14[Suppl 2]:230-235 DOI 10.1007/s10039-011-1756-6

(C) Springer-Verlag 2011

V. Kaiser

\section{Beckenfrakturen und hüftgelenknahe Verletzungen. Überlegungen zur MdE-Bewertung}

\section{Zusammenfassung}

Aufgrund der gesetzlichen Begriffskriterien ist die Bemessung der Minderung der Erwerbsfähigkeit (MdE) mit den Entwicklungen auf dem allgemeinen Arbeitsmarkt und im gesamten Erwerbsleben prinzipiell einem steten Wandel unterworfen. Somit stellt sich die Frage, ob die bisherigen MdE-Werte (noch) als allgemein gültig angesehen werden können. Insbesondere wird bezüglich der veröffentlichten Tabellenwerke diskutiert, welche dieser MdE-Sätze sich als allgemeine Erfahrungswerte qualifizieren lassen und inwieweit sie ausreichend bestimmt auf wesentliche Funktionsbeeinträchtigungen bezogen sind, um eine verlässliche Orientierung für den praktischen Einzelfall zu bieten und die notwendige Gleichbehandlung der Ver- sicherten zu gewährleisten. Abgesehen von einzelnen Entschädigungsbereichen und singulären Gesundheitsschäden erfolgten bisher keine breit angelegten Überprüfungen. Ein aufwendiges MdE-Projekt wurde jedoch kürzlich in der Kommission Gutachten, die in den orthopädischen und unfallchirurgischen Fachgesellschaften verankert ist, gestartet. Die von einer medizinisch und juristisch besetzten Arbeitsgruppe entwickelten ersten Überlegungen, v. a. zur MdE bei Beckenverletzungen, werden vorgestellt.

\section{Schlüsselwörter}

Arbeitsmarkt · MdE · Allgemeine

Erfahrungswerte $\cdot$ Beckenverletzungen .

Hüftverletzungen

\section{Pelvic fractures and injuries around the hip. Considerations on assessment of reduced fitness for work}

\begin{abstract}
Based on legal descriptive criteria, assessment of reduced fitness for work (FFW) is in principle subject to constant change in view of developments in the general labor market and working life as a whole. Thus, the question arises of whether the present parameters for determining reduced FFW can (still) be considered generally valid. Especially with respect to the published reference tables, the discussion revolves around establishing which of these measures of reduced FFW can be qualified as generally acceptable empirical values and to what extent they adequately apply to significant functional impairment in order to provide reliable orientation for practical purposes in particular cases and to ensure the necessary equal treatment
\end{abstract}

of those insured. Apart from individual areas of compensation and singular cases of health damage, no wide-ranging appraisal has been performed. A large-scale project on reduced FFW was recently initiated in the Commission on Expert Opinion which is anchored in the specialty societies for orthopedics and trauma surgery. Initial considerations formulated by a working group composed of medical and legal participants are presented, primarily regarding reduced FFW in cases of pelvic injuries.

\section{Keywords}

Employment · Reduced fitness for work . General empirical values · Pelvis, injuries . Hip injuries gebracht, zur Information über den aktuellen Stand und für Entscheidungen über die weitere Entwicklung des Projekts sowie zwischenzeitliche Äußerungen bzw. Stellungnahmen zu einzelnen Vorschlägen. Auf der Grundlage der Aufbereitungen der MdE-Arbeitsgruppe wird die Kommission das Gesamtprojekt diskutieren und die fraglichen MdE-Werte selbst prüfen; diese Projektphase schließt dann mit einer ausdrücklichen Verabschie- dung der inhaltlichen Resultate als Kommissionsvorschläge ab. Zugleich soll dann auch das weitere Prozedere gemäß der allgemeinen Zielsetzung des Projekts näher festgelegt werden.

Das MdE-Screening der ersten Projektstufe ergab vielfache Ansätze für eine nähere Überprüfung und Aktualisierung von MdE-Werten, und zwar hinsichtlich definitiver Festlegungen bei divergierenden Veröffentlichungen sowie v. a. neuer 


\begin{tabular}{|c|c|c|}
\hline \multicolumn{2}{|c|}{ Unfallfolgezustände bzw. Störungsbilder } & \multirow{2}{*}{$\begin{array}{l}\text { MdE (\%) } \\
30 \\
\end{array}$} \\
\hline Eckwerte & Instabile Verschiebung einer Beckenhälfte um 20-30 mm & \\
\hline & Stabile Verschiebung einer Beckenhälfte um 20-30 mm & 20 \\
\hline & $\begin{array}{l}\text { Erweiterung der Schambeinfuge über } 15 \mathrm{~mm} \text { (mit Beteiligung der } \\
\text { beiden Kreuz- und Darmbeinfugen), stabil verheilt }\end{array}$ & 20 \\
\hline \multirow{3}{*}{$\begin{array}{l}\text { Fremde } \\
\text { Bewertungen } \\
\text { zum Vergleich }\end{array}$} & Stabile Verschiebung einer Beckenhälfte über 10 mm [4] & 20 \\
\hline & Mehrfache Beckenringbrüche [19] ${ }^{a}$ & $30-40$ \\
\hline & Beckenbrüche mit groben Verschiebungen der Beckenhälften [19] ${ }^{a}$ & $30-40$ \\
\hline \multirow[t]{3}{*}{ Einzelwerte } & Stabile Verschiebung einer Beckenhälfte bis $10 \mathrm{~mm}$ & $<10$ \\
\hline & Erweiterung der Schambeinfuge unter $15 \mathrm{~mm}$, stabil verheilt & $<10$ \\
\hline & $\begin{array}{l}\text { Fest knöchern ausgeheilter Bruch des Beckenrings, ohne wesentliche } \\
\text { Deformation }\end{array}$ & $<10$ \\
\hline \multirow{3}{*}{$\begin{array}{l}\text { Fremde } \\
\text { Bewertungen } \\
\text { zum Vergleich }\end{array}$} & Symphysendiastase unter $15 \mathrm{~mm}$ oder -versteifung $[24]^{\mathrm{b}}[25]$ & 10 \\
\hline & $\begin{array}{l}\text { Vordere Beckenringfraktur, mäßig dislozierte Darmbeinschaufel- } \\
\text { fraktur mit Beteiligung des Kreuzbeins, knöchern fest (Bayerisches } \\
\text { LSG vom 17.07.2007) }\end{array}$ & $<10$ \\
\hline & Stabiler Beckenring mit degenerativen Veränderungen & $10(\mathrm{GdS})$ \\
\hline
\end{tabular}

Tab. 2 Literatur-MdE-Werte für sonstige Schäden/Funktionsbeeinträchtigungen des Beckens

\begin{tabular}{|c|c|c|c|}
\hline \multicolumn{2}{|c|}{ Schäden/Funktionsbeeinträchtigungen im Bereich des Beckens } & Quelle & MdE (\%) \\
\hline \multicolumn{2}{|l|}{ Stabile Beckenringfraktur mit Schoßfugen } & {$[24]^{\mathrm{a}}[25]$} & 10 \\
\hline \multicolumn{2}{|c|}{ Instabile Beckenringfraktur mit Schoßfugenerweiterung über $15 \mathrm{~mm}$} & {$[24]^{\mathrm{a}}$} & 20 \\
\hline \multicolumn{2}{|l|}{$\begin{array}{l}\text { Instabile Beckenringfraktur mit einseitiger Verschiebung } \\
\text { einer Beckenhälfte über } 10 \mathrm{~mm}\end{array}$} & {$[25]$} & 20 \\
\hline \multirow{2}{*}{$\begin{array}{l}\text { Instabile Beckenringfraktur mit beidseitiger } \\
\text { Verschiebung einer Beckenhälfte, jeweils über } 10 \mathrm{~mm}\end{array}$} & Ohne Arthrose & {$[24]^{\mathrm{a}}$} & 20 \\
\hline & Mit Arthrose & {$[25]$} & 30 \\
\hline
\end{tabular}

Tab. 3 MdE-Vorschläge zu Beeinträchtigungen im Hüftgelenk

\begin{tabular}{|lll}
\hline Beeinträchtigung im Hüftgelenk & $\begin{array}{l}\text { MdE bisher } \\
\text { (\%) }\end{array}$ & $\begin{array}{l}\text { MdE } \\
\text { (\%) }\end{array}$ \\
\hline Verlust oder vollständige Lähmung beider Beine im Hüftgelenk & Meist 100 & 70 \\
\hline $\begin{array}{l}\text { Verlust oder vollständige Gebrauchsunfähigkeit eines Beines } \\
\text { im Hüftgelenk }\end{array}$ & Meist 70-80 & 60 \\
\hline Versteifung beider Hüftgelenke in Funktionsstellung & Meist 60-100 & 60 \\
\hline Versteifung eines Hüftgelenks in ungünstiger Stellung & Meist 40-50 & $30-40$ \\
\hline Arthrose eines Hüftgelenks (Koxarthrose) mit Gebrauchsbeeinträchtigung & Meist 40 & $10-25$ \\
\hline
\end{tabular}

Vorschläge gegenüber der bisherigen Anwendungspraxis. Deshalb werden auch nicht nur selektive MdE-Vorschläge erarbeitet, sondern Arbeitsziel ist ein umfassendes Tabellenwerk, unter vorwiegender Berücksichtigung der verbreiteten aktuellen Literatur. Bis zum Frühjahr 2011 wurde von der MdE-Arbeitsgruppe ein Grundsatzpapier erstellt, mit dem die Sinnhaftigkeit des MdE-Projekts begründet wird und die entscheidenden Grundlagen für die einzelnen MdE-Vorschläge dargestellt werden sollen. Außerdem sind die Teiltabellen,

\section{Neuere Aspekte für aktuelle MdE-Werte}

Neben den bereits aufgezeigten allgemeineren Entwicklungen kristallisierten sich in jüngster Zeit neuere Aspekte heraus, die bei einer Revision des MdE-Wertgefüges - unbeachtlich ihrer unterschiedlichen Bedeutung für die Bemessung der MdE - zu berücksichtigen sind. Vorwiegend handelt es sich um spezifische Elemente in der DGUV bzw. deren CaseManagements, die sich - auch soweit sie medizinisch ausgerichtet sind - unmittelbar auf die Beschäftigungsverhältnisse und Arbeitsmöglichkeiten beziehen und dabei z. B. unmittelbar auf das berufliche Reintegrationspotenzial Unfallverletzter einwirken.

Es ist zwischenzeitlich Standard, dass die für Erwerbstätigkeiten relevanten Funktionsstörungen mit einer darauf ausgerichteten (medizinisch-gesundheitlichen) Leistungsdiagnostik detailliert erfasst werden; die hierzu entwickelten umfangreichen, insbesondere auch in den BG-Kliniken (BG: Berufsgenossenschaft) und anderen kompetenten Einrichtungen eingesetzten Instrumente schließen u. a. Assessmenttechniken und praktische Tätigkeitserprobungen ein. Diese ausgeprägte zielorientierte Blickrichtung wird auch mit einem unmittelbar arbeitsplatz- und berufsbezogenen Therapiekonzept weiter verfolgt und ist der Kern des ganzheitlichen Rehabilitationsmanagements der berufsgenossenschaftlichen Praxis; implementiert sind z. B. ärztlich kontrollierte Trainingsmaßnahmen und eine medizinische Begleitung gestufter Wiedereingliederungsversuche im Betrieb, jeweils mit enger Steuerung durch den zuständigen Unfallversicherungsträger.

Ergänzt werden alle diese intensivierten, für eine Erfolgssicherung stark strukturierten, medizinisch ausgerichteten Betreuungen des Unfallverletzten durch ein umfassendes Reintegrationsmanagement zur alsbaldigen und v. a. dauerhaften Wiederaufnahme der (regulären) Arbeit. Im SGB IX wurden die bereits bisher im Bereich der gesetzlichen Unfallversicherung breit eingesetzten berufshelferischen Aktivitäten mit dem betrieblichen Wiedereingliederungsmanagement ( $\$ 84$ Abs. 2) aufgegriffen und dabei auch dem Arbeit- 
geber konkrete Aufgaben zugewiesen, mit entsprechenden Mitwirkungspflichten anderer Stellen. Nicht zuletzt spielt im MdE-Kontext das in den Betrieben strukturiert installierte Arbeitssicherheit- und Gesundheitsmanagement eine beachtliche Rolle, ebenfalls auf verbindlichen Rechtsgrundlagen und mit reglementierenden Zuständigkeiten der Unfallversicherungsträger; es verfolgt v. a. ein präventives Erfassen von Gesundheitsrisiken zur Sicherung der Beschäftigungsfähigkeit [z. B. $\$ \$ 14$ ff. SGB VII, $\$ 5$ Arbeitsschutzgesetz, BG-Vorschriften (BGV) und -Richtlinien (BGR)]. Diese neuere Entwicklung wird begleitet von einer allgemein sensibleren Unternehmenskultur gegenüber gesundheitlich beeinträchtigten bzw. leistungsgeminderten Mitarbeitern, mitsamt einer höheren Aufgeschlossenheit der Belegschaft gegenüber solchen Kollegen, ungeachtet der Anforderungen der modernen Arbeitswelt an den Einzelnen $[7,18]$.

Für die aktuelle Rechtsprechungspraxis ist eine zunehmende Orientierung der individuellen MdE am tatsächlichen Ausmaß der verbliebenen Erwerbsmöglichkeiten und unter Berücksichtigung des gesamten, konkreten Rehabilitationsergebnisses bemerkenswert. So wurde z. B. in einer Entscheidung des LSG (Landessozialgericht) Berlin-Brandenburg vom 23.09.2009 auf die allgemeine Mobilität und Bewegungsfreiheit beim querschnittgelähmten Unfallversicherten abgestellt und die MdE im Hinblick auf dessen Fähigkeit zum selbstständigen Autofahren - im Gegensatz zur Vorinstanz und der bisher üblichen Höhe von 100\% - auf $90 \%$ festgesetzt.

\section{MdE-Vorschläge: \\ Beckenverletzungen}

Entsprechend ihrer allgemeinen Zielsetzung konzentrierte sich die MdE-Arbeitsgruppe, jedenfalls mit ihrem bisherigen Arbeitsergebnis, auf einige wesentliche Funktionsbeeinträchtigungen und knüpfte dabei auch nicht speziell an die Beckenrand- und Hüftpfannenfrakturen an. AuBerdem sollten solche Unfallfolgezustände aufgelistet werden, denen sich - entgegen den gerade diesbezüglich in den Literaturvorschlägen üblichen Spreizungen
- eindeutige MdE-Werte zuordnen lassen; aus diesen festen Sätzen können wiederum Einschätzungen für Funktionsausfälle abgeleitet werden. Im Übrigen ist ein Vergleich der verschiedenen veröffentlichten MdE-Tabellen zum Teil wegen der unterschiedlichen Beschreibung ihrer Schadensbilder bzw. Bezugspunkte schwierig.

Ungeachtet dessen lassen sich in der einschlägigen Literatur $[2,3,4,8,10,19$, $22,24,25]$ weit gehend folgende Grundorientierungen feststellen, denen sich die MdE-Arbeitsgruppe anschloss: Stabile Beckenfrakturen führen in der Regel zu einer MdE unter 10\% (keine messbare MdE); ausgenommen sind Unfallfolgezustände mit stärkerer Diastase bzw. Verschiebung einer Beckenhälfte. Instabil verheilte Beckenfrakturen führen regelmäßig zu einer MdE von wenigstens $20 \%$. Aber alle auf isolierte unfallchirurgisch-orthopädische Schadensbilder bzw. entsprechende Funktionsstörungen ausgerichtete MdEVorschläge können für die individuelle MdE-Einschätzung im praktischen Einzelfall insoweit nur eine beschränkte Bemessungshilfe geben, als Beckenbrüche zumeist mit Mehrfachverletzungen (insbesondere auf urologischem, neurologischem und gefäßmedizinischem Gebiet) assoziiert sind. Mit diesen Kautelen hält die MdE-Arbeitsgruppe die in $\mathbf{0}$ Tab. 1 aufgeführten MdE-Werte für angemessen, verteilt auf Eckwerte für grundlegende, als Markierungen im MdE-Gefüge geeignete Unfallfolgezustände bzw. Störungsbilder und Einzelwerte, die sich darin einordnen:

In der Literatur finden sich für sonstige Schäden bzw. Funktionsbeeinträchtigungen im Bereich des Beckens u. a. noch die in $\square$ Tab. 2 aufgeführten MdE-Bewertungen.

\section{Exkurs}

\section{MdE-Vorschläge zu Beeinträchtigungen im Hüftgelenk}

Zur Einordnung der MdE-Vorschläge für Beckenverletzungen werden die Ergebnisse der MdE-Arbeitsgruppe zum Hüftgelenk (in dem Tabellenwerk untere Extremitäten) wiedergegeben, die teilweise markant von bisherigen Tabellen in der Literatur abweichen $[3,22,25]$. Sie können insbesondere im Hinblick auf den dargestellten Ablauf des MdE-Projekts nur als ein erster Meinungsstand angesehen werden, v. a. auch weil sich die Kommission Gutachten der DGOU insgesamt noch nicht damit näher befasste. Die in - Tab. 3 aufgeführten Vorschläge sollen den skizzierten Entwicklungen in der allgemeinen Arbeitsgestaltung sowie den grundlegenden Beschäftigungsbedingungen Rechnung tragen und die aktuellen betrieblichen Gegebenheiten und Standards im Erwerbsleben widerspiegeln.

\section{Überlegungen zu den oberen Extremitäten}

Die MdE-Bemessungen für die oberen Extremitäten wurden von der $M d E$ Arbeitsgruppe noch nicht im Einzelnen untersucht. Leitaspekt dafür wird aber, entsprechend den für aktuelle MdE-Sätze relevanten Faktoren, eine größere Bedeutung der oberen Extremitäten sein; dies kommt besonders im Vergleich zu den unteren Extremitäten zum Ausdruck, für deren Einschätzung von MdE-Graden z. B. der allgemeine Rückgang stehender Tätigkeiten und das Bewegen schwerer Gegenstände kennzeichnend sind. Daraus ergibt sich eine breite Tendenz zu höheren MdE-Werten, als sie aktuell (noch) gängig sind; v. a. für Funktionsbeeinträchtigungen der Hände werden solche Überlegungen von Bedeutung sein.

\section{Korrespondenzadresse}

\section{Dr. V. Kaiser}

Hauffstraße 16,

71120 Grafenau

Dr.Volker.Kaiser@t-online.de

Interessenkonflikt. Der korrespondierende Autor gibt an, dass kein Interessenkonflikt besteht.

\section{Literatur}

1. Becker J (2008) Aktuelle Fragen zur Beurteilung von Implantaten und Prothesen. Med Sach 105:1425

2. Beickert R (2008) Begutachtung von Beckenverletzungen. Trauma Berufskrankh 10(2):147-154

3. Bereiter-Hahn W, Mehrtens G (2011) Gesetzliche Unfallversicherung, Handkommentar, 5. Aufl. Schmidt, Berlin 
4. Echtermeyer V, Sangmeister M, Lange K (1995) Begutachtungen nach Beckenverletzungen aus chirurgischer Sicht. In: Hierholzer G, Kunze G, Peters D (Hrsg) Gutachtenkolloquium 10. Springer, Berlin Heidelberg New York, S 127

5. Elsner G, Seidler A (2003) Zur Frage der evidenzbasierten Bewertung der Minderung der Erwerbsfähigkeit. Zentralbl Arbeitsmed 53:442

6. Foerster K, Bork S, Kaiser V et al (2007) Vorschläge zur MdE-Einschätzung bei psychoreaktiven Störungen in der gesetzlichen Unfallversicherung. Med Sach 103:52

7. Fornoff CH, Schmickal Th, Ritter F (2006) Rehabilitationsmanagement. Trauma Berufskrankh [Suppl 1] 8:S93-S104

8. Hutschenreiter G, Fahle H (1995) Begutachtungen nach Beckenverletzungen aus urologischer Sicht. In: Hierholzer G, Kunze G, Peters D (Hrsg) Gutachtenkolloqium 10. Springer, Berlin Heidelberg New York, 135

9. Kaiser V (1995) Einschätzung der Minderung der Erwerbsfähigkeit nach Therapiewirkung: Sind mittelbar-funktionelle Behandlungserfolge relevant? Aktuelle Traumatol 25:202

10. Kaiser V (2000) Begutachtung von Verletzungen des Beckens und der Hüfte: Aktuelle Hinweise mit Praxisfällen zur MdE. Aktuelle Traumatol 30:108

11. Kaiser V (2006) Rechtliche Bedeutung von „Leitlinien" und anderer qualifizierter Texte in der Begutachtung. Forum 19

12. Keller W (2002) Die MdE bei Berufskrebserkrankungen im Unfallversicherungsrecht. Med Sach 98:13

13. Keller W (2003) Das antizipierte Sachverständigengutachten im Sozialrecht. SGb 254

14. Keller W (2006) Erfahrungen mit antizipierten Sachverständigengutachten im Berufskrankheitenrecht. MedSach 102:128

15. Kranig A (2001) Grundsatzfragen zur Rentenbemessung nach dem Grad der Minderung der Erwerbsfähigkeit (MdE). Sozial Fortschr 113

16. Kranig A (2010) Bedeutung von Begutachtungsempfehlungen, antizipierten Sachverständigengutachten und Leitlinien - aus der Sicht der gesetzlichen Unfallversicherung. MedSach 106:54

17. Meier H (Hrsg) (1990) Zur Diagnose der Moderne. Piper, München

18. Mehrhoff F (Hrsg) (2004) Disability Management. Strategien zur Integration behinderter Menschen in das Arbeitsleben, 1. Aufl. Gentner, Stuttgart

19. Mehrhoff F, MeindI RC, Muhr G (2010) Unfallbegutachtung, 12. Aufl. deGruyter, Berlin

20. Plagemann $\mathrm{H}$ (2004) Muss eine Funktionsverbesserung durch Heil- und Hilfsmittel sich auf die MdE-/GdB-Beurteilung auswirken? - Aus Sicht eines Anwalts. Med Sach 100:94

21. Ricke W (1989) Arbeitsplatzstatistiken und Minderung der Erwerbsfähigkeit: Neue Wege zur MdE oder des Kaisers neue Kleider? Die BG 288

22. Rompe G, Erlenkämper A, Schiltenwolf M, Hollo DF (2009) Begutachtung der Haltungs- und Bewegungsorgane, 5. Aufl. Thieme, Stuttgart New York

23. Rosa H (2005) Beschleunigung. Die Veränderung der Zeitstrukturen in der Moderne. Suhrkamp, Frankfurt am Main

24. Schönberger A, Mehrtens G, Valentin H (2010) Arbeitsunfall und Berufskrankheit, 8. Aufl. Schmidt, Berlin

25. Thomann KD, Schröter F, Grosser V (2009) Handbuch der orthopädisch-unfallchirurgischen Begutachtung, 1. Aufl. Urban \& Fischer, München

26. Wiester W (2001) Über die Aufgabe der Unfallversicherungsträger, die Richtwerte zur Bemessung der MdE überprüfen zu lassen. NZS 630 